\title{
Molecular Insight on the Molecular Mechanisms for the Biological Activity of Dendritic Amphiphiles on Biological Membranes
}

四VirginiaTech

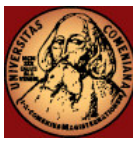

\section{A. K. Sum ${ }^{1}$, R. D. Gandour ${ }^{2}$, J. Karlovská3 ${ }^{3}$ S. S. Funari, P. Balgavý3}

${ }^{1}$ Department of Chemical Engineering, ${ }^{2}$ Department of Chemistry, Virginia Polytechnic Institute and State University, Blacksburg, Virginia, USA

${ }^{3}$ Department of Physical Chemistry of Drugs, Faculty of Pharmacy, Comenius University, Odbojárov 10, SK-832 32 Bratislava, SLOVAKIA 48th International Conference on the

Bioscience of Lipids

Turku, FINLAND

4-8 September, 2007

\section{Introduction}

Lipid bilayers are central in the study and understanding of biological membranes. Their structure, function, and activity are directly related to the composition and molecular properties of the membrane constituents. In an effort to understand the antimicrobial activity of a new class of dendritic amphiphiles (3CAmn)-potentially safe, effective, acceptable, and affordable topical microbicides with anti-HIV, antiSTD pathogens, antibacterial, and antifungal activities [1], we combine experimental and computational techniques to obtain insight into how these amphiphiles interact with lipid bilayers. Because 3CAmn are lipid-like compounds, their interactions with the lipid bilayer of biological membranes result in biophysical changes in the structure and dynamics of the membranes. Wide-angle (WAXD) and small-angle (SAXD) X-ray diffraction on model DPPC membranes show systematic changes to the membrane structure and, consequently, the phase transition due to interaction with these dendritic amphiphiles. Molecular dynamics simulations of these structures reveal the specific molecular mechanisms associated with the changes to the membrane structure. This study represents a multi-scale modeling approach to design, develop, and understand the biological activities of this homologous series of dendritic amphiphiles that include very long chains. [1] Williams, A. A.; Sugandhi, E. W.; Macri, R. V.; Falkinham, J. O., III; Gandour, R. D. Antimicrobial Activity of Long-Chain, Water-
Soluble, Dendritic Tricarboxylato Amphiphiles. J. Antimicrob. Chemother. 51, 451-458 (2007).

Dendritic Tricarboxyl Amphiphiles

Dipalmitoylphosphatidylcholine (DPPC)

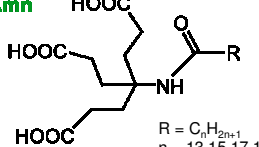

$R=C_{n} H_{2 n+1}, 1,21,23$
$n=13,15,17,19,21$,

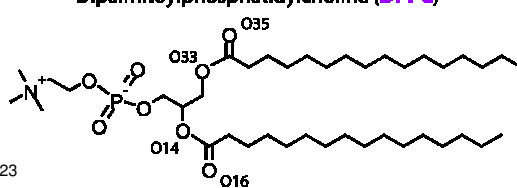

3CAmn HOOC

\section{Experimental: Materials and Methods}

Long chained amphiphile and DPPC (Avanti) were mixed in desired molar ratio in chloroform + methanol, the solvent was evaporated under a stream of gaseous nitrogen and its traces removed by an oil vacuum pump. Redistilled water was added at the $\mathrm{H}_{2} \mathrm{O}: \mathrm{DPPC}=5: 1$ weight ratio and the mixture was homogenized mechanically at $60{ }^{\circ} \mathrm{C}$. SAXD and WAXD experiments were performed at beam A2 in HASYLAB at DESY using a monochromatic radiation of wavelength $0,15 \mathrm{~nm}$. During temperature scans, the samples were heated from 20 to $60{ }^{\circ} \mathrm{C}$ in $40 \mathrm{~min}$. and the diffractograms were recorded for $10 \mathrm{sec}$. every min. Data reduction, normalization and analysis were done with the programs STAFO, OTOKO, PEAKFIT and GRAPHER.

\section{Simulation Methods}

NPT simulations

Berendsen thermostat

Berendsen barostat

2 fs time-step

Over 80 ns of simulation tim

Short-range cutoff: $1.0 \mathrm{~nm}$

PME for long-range electrostatics

All-bonds constrained (LINCS and SHAKE)

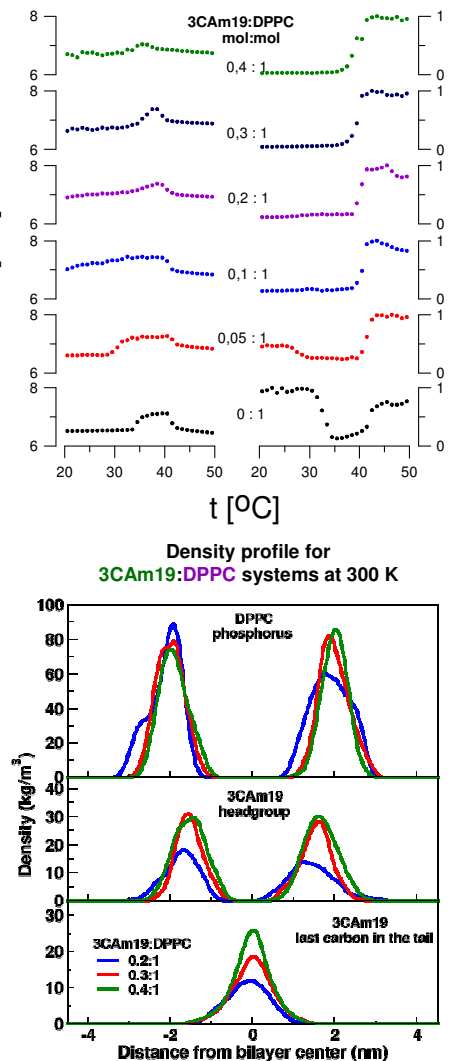

$\rightarrow$ Diffraction peaks fitted with Lorentzians and linear background to determine maxima, intensities, integral intensities, and half-width of peaks

$\rightarrow$ Pre-transition and main transition were present for pure DPPC and 0.05:1 3CAm19:DPPC

$\rightarrow$ Pre-transition was shifted to lower temperature for the mixtures

$\rightarrow$ Data used to obtain main gel-liquid crystalline phase transition temperature

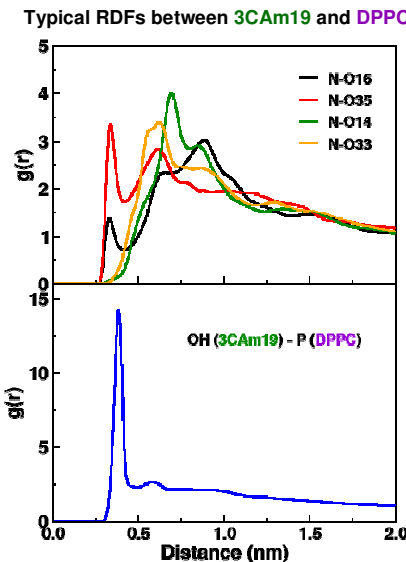

$\rightarrow 3 \mathrm{CAm} 19$ amide group hydrogen bonds to DPPC carbonyl group, mainly O35

$\rightarrow 3$ CAm19 acid groups hydrogen bond to DPPC phosphate headgroup
Water: SPC model

DPPC: Berger et al. and Chiu et al.

3CAmn: OPLS

United-atom for methyl and methylene groups GROMACS 3.3.1 (single precision in parallel)

\section{CAm19:DPPC $\quad 3$ 3CAm19 $\quad$ DPPC $\quad$ All systems simulated at

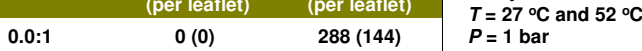

$0.2: 1 \quad 48(24) \quad 240(120)$

0.3:1 $66(33) \quad 222(111)$

0.4:1 $82(41) \quad 206(103)$

All systems contained $\mathbf{4 0}$ water per total 3CAm19+ DPPC
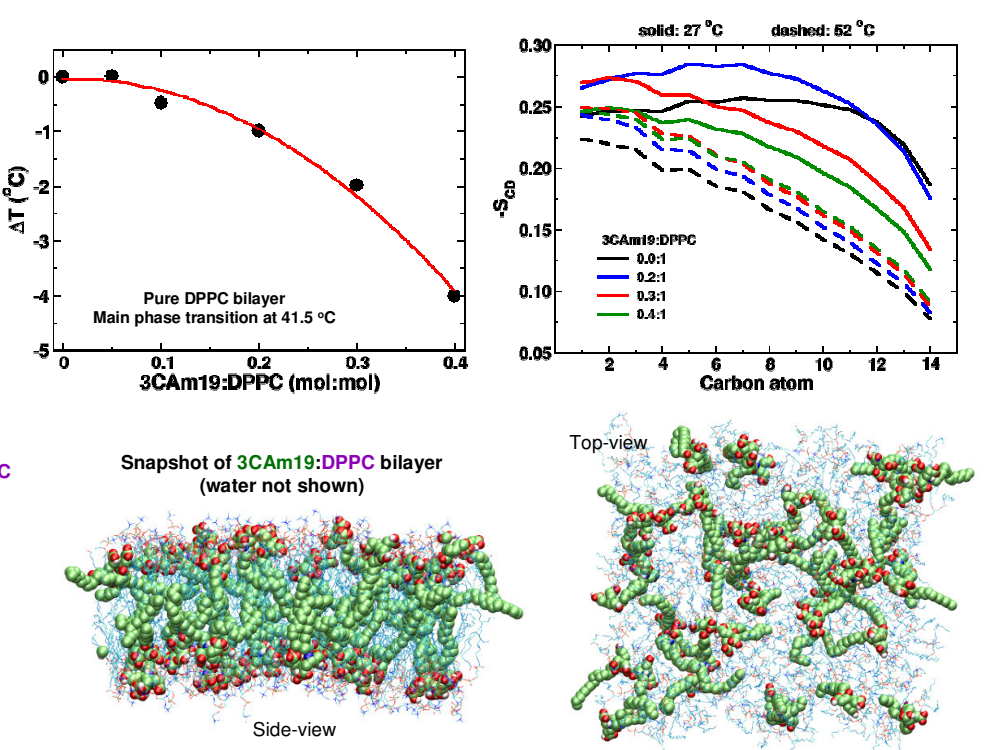

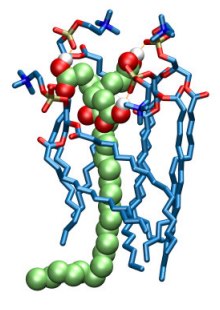

Liquidcrystalline state $\rightarrow 3 \mathrm{CAm} 19$ aligns with lipid along the bilaye $\rightarrow$ At $325 \mathrm{~K}, 3 \mathrm{CAm} 19$ induces a slight order in the bilaye $\rightarrow$ At $300 \mathrm{~K}, 3 \mathrm{CAm} 19$ induces significant order on the carbon atoms near the headgroups

$\rightarrow$ In the liquid-crystalline state, most 3CAm19 molecules have the end tail bent

$\rightarrow$ In the gel state, most 3CAm19 molecules have the end tail straight penetrating the opposing leaflet

$\rightarrow$ On average, four DPPC molecules surround each 3CAm19

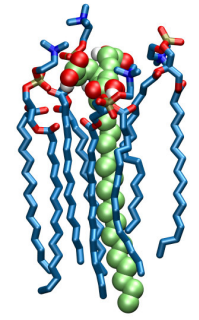

Gel state
The experimental portion of this study was supported by the European Community - Research Infrastructure Action under the FP6 "Structuring the European Research Area" Programme Contract RII3-CT-2004-506008 (HASYLAB project II-05-018 EC) and by the VEGA 1/3029/06 and APVV 51-027404 grants.
- Beam-time for SAXD and WAXD experiments made available in HASYLAB at DESY

- Computational resources provided by Virginia Tech Advanced Computing Research facilities

- Travel financial assistance provided by VT Chemical Engineering and VT Research Office 